\title{
History states of systems and operators
}

\author{
A. Boette ${ }^{1}$ and R. Rossignoli ${ }^{1,2}$ \\ ${ }^{1}$ Departamento de Física-IFLP/CONICET, Universidad Nacional de La Plata, C.C. 67, La Plata (1900), Argentina \\ ${ }^{2}$ Comisión de Investigaciones Científicas (CIC), La Plata (1900), Argentina
}

\begin{abstract}
We discuss some fundamental properties of discrete system-time history states. Such states arise for a quantum reference clock of finite dimension and lead to a unitary evolution of system states when satisfying a static discrete Wheeler-DeWitt-type equation. We consider the general case where system-clock pairs can interact, analyzing first their different representations and showing there is always a special clock basis for which the evolution for a given initial state can be described by a constant Hamiltonian $H$. It is also shown, however, that when the evolution operators form a complete orthogonal set, the history state is maximally entangled for any initial state, as opposed to the case of a constant $H$, and can be generated through a simple double-clock setting. We then examine the quadratic system-time entanglement entropy, providing an analytic evaluation and showing it satisfies strict upper and lower bounds determined by the energy spread and the geodesic evolution connecting the initial and final states. We finally show that the unitary operator that generates the history state can itself be considered as an operator history state, whose quadratic entanglement entropy determines its entangling power. Simple measurements on the clock enable to efficiently determine overlaps between system states and also evolution operators at any two times.
\end{abstract}

\section{INTRODUCTION}

The incorporation of time in a fully quantum framework [1] has recently attracted wide attention 2 11. On the one hand, it is relevant as a fundamental problem and a key issue in the search for a coherent theory of quantum gravity 12 18]. On the other hand, a quantum description of time enables to exploit the quantum features of superposition and entanglement in the development of new models of parallel-in-time simulation [6, 7].

The concept of time is related to the quantification of evolution through a reference physical system called clock. Historically, the readings of this clock provided an external classical parameter, called time. Nonetheless, if we aim to introduce time into a fully quantum framework, the clock has to be a quantum system itself. This is even more important in attempts to quantize gravity where time has to be described by a dynamical entity 13 18].

Here we describe the system and the reference clock through a discrete system-time history state which enforces a discrete unitary evolution on the system states. We consider the general case where the system-clock pairs can interact. This scenario provides a more general starting point, more adequate for some quantum gravity or cosmological models where interactions between an internal relational clock and evolving degrees of freedom cannot be excluded 17, 18].

We first discuss different representations of the history state, showing that for a fixed initial state there is always an adequate selection of clock basis for which the resultant evolution corresponds to a constant Hamiltonian, with the history state satisfying a discrete counterpart of a standard Wheeler-DeWitt type equation [12. The general interacting formalism opens, however, new possibilities. The entanglement of the history state is a measure of the number of orthogonal states visited by the system at orthogonal times [7, and for a constant Hamiltonian clearly depends on the seed system state. This depen- dence becomes, however, attenuated when the Hamiltonian is not constant in time, and in the case where the evolution operators form a complete orthogonal set, it is in fact always maximum, irrespective of the initial state. The corresponding history state admits, nonetheless, a simple generation through a two-clock scenario, where the clocks are linked to conjugate system variables.

We then analyze the quadratic entanglement entropy of history states, which, as opposed to the standard entropy, can be explicitly evaluated in the general case, enabling one to characterize the system evolution and also to connect the entanglement of states and operators. For a general constant Hamiltonian it can be analytically determined for any number of steps. Moreover, we show that it is upper bounded by the quadratic entropy of the energy spread of the initial state and lower bounded by that of the geodesic evolution connecting the initial and final states according to the Fubini-Study metric [19]. And its average over all initial system states is directly proportional to the quadratic operator entanglement entropy 20 23] of the unitary gate that generates the history state. Through the channel-state duality 24 28], it is also shown that the pure state which represents the latter is itself an operator history state, whose quadratic entanglement entropy determines its entangling power.

Finally, we show that through measurements on the clock it is possible to use both system and operator history states to efficiently determine the overlap between system states and also the trace of the evolution operator between any two-times. The latter reduces to the trace of a unitary operator (result of the DQC1 circuit 29 ) for the simple case of a qubit clock. The properties of general discrete history states and their entanglement are discussed in section [II whereas the entanglement and history states of unitary operators are discussed in III. Conclusions are finally given in IV 


\section{DISCRETE HISTORY STATES}

We consider a system $S$ and a reference clock system $T$ in a joint pure state $|\Psi\rangle \in \mathcal{H}_{S} \otimes \mathcal{H}_{T}$, with $\mathcal{H}_{T}$ of finite dimension $N$. Any such state can be written as

$$
|\Psi\rangle=\frac{1}{\sqrt{N}} \sum_{t}\left|S_{t}\right\rangle|t\rangle
$$

where $|t\rangle, t=0, \ldots, N-1$, are orthogonal states of $T$ $\left(\left\langle t \mid t^{\prime}\right\rangle=\delta_{t t^{\prime}}\right)$ and $\left|S_{t}\right\rangle$ are states of $S$, not necessarily orthogonal or normalized, yet satisfying $\sum_{t}\left\langle S_{t} \mid S_{t}\right\rangle / N=$ $\langle\Psi \mid \Psi\rangle=1$. Consider now a unitary operator $\mathcal{U}$ for the whole system of the form

$$
\mathcal{U}=\sum_{t=1}^{N} U_{t, t-1} \otimes|t\rangle\langle t-1|
$$

where $t=N$ is identified with $t=0$ and $U_{t, t-1}$ are arbitrary unitary operators on $S$ satisfying $U_{0, N-1} \ldots U_{1,0}=$ $\mathbb{1}$. If $|\Psi\rangle$ fulfills the eigenvalue equation

$$
\mathcal{U}|\Psi\rangle=|\Psi\rangle
$$

the states $\left|S_{t}\right\rangle$ will undergo a unitary evolution with $t$ :

$$
\begin{aligned}
\left|S_{t}\right\rangle=\sqrt{N}\langle t \mid \Psi\rangle & =\sqrt{N}\langle t|\mathcal{U}| \Psi\rangle \\
& =U_{t, t-1}\left|S_{t-1}\right\rangle=U_{t}\left|S_{0}\right\rangle,
\end{aligned}
$$

where $U_{t}=U_{t, t-1} \ldots U_{1,0}$, with $U_{0}=\mathbb{1}$. The states $\left|S_{t}\right\rangle$ will then have a unit norm if $|\Psi\rangle$ is normalized.

Thus, the state (1) is a discrete finite dimensional version of the history state of the Page-Wootters formalism [1, 3]. Moreover, writing $\mathcal{U}=\exp [-i \mathcal{J}]$, with $\mathcal{J}$ hermitian (and spectrum $\subset[0,2 \pi)$ ), Eq. (3) is equivalent to

$$
\mathcal{J}|\Psi\rangle=0
$$

which is a discrete cyclic version of a Wheeler-DeWitt type equation [12]. Note, however, that $\mathcal{J}$ will contain $S-T$ interaction terms in the general case where $U_{t, t-1}$ depends on $t$.

A unitary evolution of the states $\left|S_{t}\right\rangle$ actually occurs if $|\Psi\rangle$ is any eigenstate of $\mathcal{U}$ : Its eigenvalues are $e^{-i 2 \pi k / N}$, $k=0, \ldots, N-1$, and its eigenstates have all the form (1) with $\left|S_{t}\right\rangle$ satisfying a shifted unitary evolution: $\left|S_{t}\right\rangle=$ $e^{i 2 \pi k / N} U_{t, t-1}\left|S_{t-1}\right\rangle=e^{i 2 \pi k t / N} U_{t}\left|S_{0}\right\rangle$. Each eigenvalue has degeneracy equal to the dimension $d_{S}=\operatorname{dim} \mathcal{H}_{S}$ of the system space, with its eigenspace spanned by orthogonal history states $\left|\Psi_{k}^{l}\right\rangle$ generated by $d_{S}$ orthogonal initial states $\left|S_{0}^{l}\right\rangle:\left\langle\Psi_{k}^{l} \mid \Psi_{k^{\prime}}^{l^{\prime}}\right\rangle=\left\langle S_{0}^{l} \mid S_{0}^{l^{\prime}}\right\rangle=\delta^{l l^{\prime}}$ [].

If $U_{t, t-1}$ is independent of $t \forall t=1, \ldots, N$, then

$$
U_{t, t-1}=\exp \left[-i H_{S}\right]
$$

with $H_{S}$ a fixed hermitian Hamiltonian for system $S$ with eigenvalues $2 \pi k / N, k$ integer. The operator (2) becomes then separable: $\mathcal{U}=\exp \left[-i H_{S}\right] \otimes \exp \left[-i P_{T}\right]$, implying

$$
\mathcal{J}=H_{S} \otimes \mathbb{1}+\mathbb{1} \otimes P_{T}
$$

which contains no interaction terms. Here $P_{T}$ is the generator of time translations, satisfying $e^{-i P_{T}}|t-1\rangle=|t\rangle \forall$ $t$ and $P_{T}|k\rangle_{T}=\frac{2 \pi k}{N}|k\rangle_{T}$, with $|k\rangle_{T}$ the discrete Fourier transform (DFT) of the states $|t\rangle$ :

$$
|k\rangle_{T}=\frac{1}{\sqrt{N}} \sum_{t} e^{i 2 \pi k t / N}|t\rangle, \quad k=0, \ldots, N-1
$$

Eqs. (5) - (7) then become an exact discrete version of the usual static Wheeler-DeWitt equation [3]. The ensuing condition $\langle t|\mathcal{J}| \Psi\rangle=0$ implies

$$
-\left\langle t\left|P_{T}\right| \Psi\right\rangle=H_{S}\left|S_{t}\right\rangle
$$

which is a discrete version of Schrödinger's equation: As $-\left\langle t\left|P_{T}\right| t^{\prime}\right\rangle=i \frac{\partial}{\partial t} \frac{1}{N} \sum_{k} e^{i 2 \pi k\left(t-t^{\prime}\right) / N}$, for $N \rightarrow \infty$ $-\left\langle t\left|P_{T}\right| t^{\prime}\right\rangle \rightarrow i \delta^{\prime}\left(t-t^{\prime}\right)$ and $-\left\langle t\left|P_{T}\right| \Psi\right\rangle \rightarrow i \frac{\partial}{\partial t}\left|S_{t}\right\rangle$.

\section{A. Representations and entanglement of the history state}

By considering an arbitrary orthogonal basis $\{|q\rangle\}$ of $\mathcal{H}_{S}$, we may first rewrite $|\Psi\rangle$ as

$$
|\Psi\rangle=\frac{1}{\sqrt{N}} \sum_{q, t} \psi(q, t)|q t\rangle
$$

where $|q t\rangle=|q\rangle|t\rangle$ and $\psi(q, t)=\left\langle q \mid S_{t}\right\rangle=\sqrt{N}\langle q t \mid \Psi\rangle$ is a "wave function" satisfying a unitary evolution with $t$ : $\psi(q, t)=\sum_{q^{\prime}}\left\langle q\left|U_{t, t-1}\right| q^{\prime}\right\rangle \psi\left(q^{\prime}, t-1\right)$.

We may then obtain the Schmidt decomposition of $|\Psi\rangle$, which we will here write as

$$
|\Psi\rangle=\sum_{k} \lambda_{k}|k\rangle_{S}|-k\rangle_{T}
$$

where $\lambda_{k}>0$ are the singular values of the matrix $\psi(q, t) / \sqrt{N}$ and $|k\rangle_{S(T)}$ orthonormal states of $S(T)$ derived from the singular value decomposition of $\psi(q, t)$, with $|-k\rangle \equiv|N-k\rangle$. They are eigenstates of the reduced states $\rho_{S(T)}=\operatorname{Tr}_{T(S)}|\Psi\rangle\langle\Psi|$, with $\lambda_{k}^{2}$ their non-zero eigenvalues. While the states $\left|S_{t}\right\rangle \propto\langle t \mid \Psi\rangle$ are not necessarily orthogonal but are equally probable, the states $|k\rangle_{S} \propto{ }_{T}\langle-k \mid \Psi\rangle$ are all orthogonal but not equally probable, with $\lambda_{k}^{2}$ representing a "permanence" probability.

In the constant case (6)-(7), the Schmidt states $|k\rangle_{S}$ and $|k\rangle_{T}$ are just the eigenstates of $H_{S}$ and $P_{T}$ :

$$
H_{S}|k\rangle_{S}=\frac{2 \pi k}{N}|k\rangle_{S}, \quad P_{T}|k\rangle_{T}=\frac{2 \pi k}{N}|k\rangle_{T}
$$

since $\left|S_{t}\right\rangle=e^{-i H_{S} t}\left|S_{0}\right\rangle=\sum_{k} \lambda_{k} e^{-i 2 \pi k t / N}|k\rangle_{S}$ with $\lambda_{k}=$ ${ }_{S}\left\langle k \mid S_{0}\right\rangle$, and hence $|\Psi\rangle=\frac{1}{\sqrt{N}} \sum_{k, t} \lambda_{k} e^{-i 2 \pi k t / N}|k\rangle_{S}|t\rangle$ becomes Eq. (11), with $|k\rangle_{T}$ the strictly orthogonal states (8). The Schmidt coefficients $\lambda_{k}$ represent in this case the distribution of $\left|S_{0}\right\rangle$ over distinct energy eigenstates (in case of degeneracy, $\lambda_{k}|k\rangle_{S}$ denotes the projection of $\left|S_{0}\right\rangle$ onto the eigenspace of energy $2 \pi k / N(\bmod 2 \pi)$, with 
$\lambda_{k}^{2}$ the total probability of measuring this energy in $\left.\left|S_{0}\right\rangle\right)$. It is then apparent from Eqs. (17) and (11) that $|\Psi\rangle$ satisfies Eq. (5), which becomes a zero "total momentum" condition: $k_{S}+k_{T}=0(\bmod N)$.

In the case of arbitrary unitary operators $U_{t, t-1}$ in (2), for any given initial state $\left|S_{0}\right\rangle$ there is always, however, a special orthogonal basis of $\mathcal{H}_{T}$ for which the corresponding states of $S$ evolve according to a constant Hamiltonian $H_{S}$ satisfying (12). It is just necessary to use the inverse DFT of the Schmidt states $|k\rangle_{T}$ of (11),

$$
|\tau\rangle=\frac{1}{\sqrt{N}} \sum_{k} e^{-i 2 \pi k \tau / N}|k\rangle_{T}
$$

with $k, \tau=0, \ldots, N-1$ (if the Schmidt rank is less than $N$, the states $|k\rangle_{T}$ of (11] can be completed with orthogonal states), which will not coincide in general with the original states $|t\rangle$. The state (11) then becomes

$$
|\Psi\rangle=\frac{1}{\sqrt{N}} \sum_{\tau, k} \lambda_{k} e^{-i 2 \pi k \tau / N}|k\rangle_{S}|\tau\rangle=\frac{1}{\sqrt{N}} \sum_{\tau}\left|S_{\tau}\right\rangle|\tau\rangle,
$$

where $\left|S_{\tau}\right\rangle=\sum_{k} e^{-i 2 \pi k \tau / N} \lambda_{k}|k\rangle_{S}$ satisfies

$$
\left|S_{\tau}\right\rangle=\sqrt{N}\langle\tau \mid \Psi\rangle=\exp \left[-i \tau H_{S}\right]\left|S_{\tau=0}\right\rangle,
$$

with $\left|S_{\tau=0}\right\rangle=\sum_{k} \lambda_{k}|k\rangle_{S}$ and $H_{S}$ defined over the Schmidt states $|k\rangle_{S}$ by Eq. (12). The Schmidt coefficients $\lambda_{k}$ can then be interpreted as the distribution of $\left|S_{\tau=0}\right\rangle$ over these energy eigenstates. In terms of the operators $H_{S}$ and $P_{T}$ defined by (12), $|\Psi\rangle$ satisfies Eq. (5) also for an effective non-interacting $\mathcal{J}$ of the form (7), and can be generated from $\left|S_{\tau=0}\right\rangle\left|0_{\tau}\right\rangle$ with the circuit of Fig. (11).

Assuming now $d_{S}=N$ (the Schmidt decomposition selects in any case subspaces of equal dimension on $S$ and $T$ ) we can also consider the inverse DFT of the system Schmidt states, $|\xi\rangle=\frac{1}{\sqrt{N}} \sum_{k} e^{-i 2 \pi k \xi / N}|k\rangle_{S}$, which satisfy $e^{-i H_{S}}|\xi\rangle=|\xi+1\rangle$ and are the special system states analogous to $|\tau\rangle$. We can then also rewrite $|\Psi\rangle$ as

$$
|\Psi\rangle=\frac{1}{\sqrt{N}} \sum_{\xi, \tau} \Lambda_{\xi-\tau}|\xi \tau\rangle=\sum_{\xi} \Lambda_{\xi}\left|\Psi_{\xi}\right\rangle
$$

where $\sqrt{N}\langle\xi \tau \mid \Psi\rangle=\Lambda_{\xi-\tau}$ depends just on $\xi-\tau$, and

$$
\Lambda_{\xi}=\frac{1}{\sqrt{N}} \sum_{k} e^{i 2 \pi k \xi / N} \lambda_{k}
$$

is the DFT of the Schmidt coefficients $\lambda_{k}$, with $\left|\Psi_{\xi}\right\rangle=$ $\frac{1}{\sqrt{N}} \sum_{\tau}|\xi+\tau\rangle|\tau\rangle$ orthogonal maximally entangled history states: $\left\langle\Psi_{\xi} \mid \Psi_{\xi^{\prime}}\right\rangle=\delta_{\xi \xi^{\prime}}(|\xi+\tau\rangle \equiv|\xi+\tau-N\rangle$ if $\xi+\tau \geq N)$.

The representation (16) is then "conjugate" to (11), expressing $|\Psi\rangle$ as a superposition of maximally entangled orthogonal history states. Like (11), it is symmetric in $S-T$ : States $\left|S_{\tau}\right\rangle=\sqrt{N}\langle\tau \mid \Psi\rangle=\sum_{\xi} \Lambda_{\xi-\tau}|\xi\rangle$ evolve unitarily with $\tau$ (Eq. (15)) while clock states $\left|T_{\xi}\right\rangle=\sqrt{N}\langle\xi \mid \Psi\rangle=\sum_{\tau} \Lambda_{\xi-\tau}|\tau\rangle$ evolve unitarily with $\xi$ :

$$
\left|T_{\xi}\right\rangle=\sqrt{N}\langle\xi \mid \Psi\rangle=\exp \left[-i \xi P_{T}\right]\left|T_{\xi=0}\right\rangle
$$

where $\left|T_{\xi=0}\right\rangle=\sum_{k} \lambda_{k}|-k\rangle_{T}$, complementing Eq. (15). Both $\xi$ and $\tau$ always run from 0 to $N-1$ with uniform weight, irrespective of the seed state.

From the Schmidt decomposition (11) we can evaluate the system-time entanglement entropy [7]

$$
E(S, T)=S\left(\rho_{S}\right)=S\left(\rho_{T}\right)=-\sum_{k} \lambda_{k}^{2} \log _{2} \lambda_{k}^{2},
$$

where $S(\rho)=-\operatorname{Tr} \rho \log _{2} \rho$. If $\left|S_{0}\right\rangle$ happens to be a common eigenstate of all $U_{t, t-1}$, such that $\left|S_{t}\right\rangle=e^{-i \phi_{t}}\left|S_{0}\right\rangle$ $\forall t$, then $|\Psi\rangle \propto\left|S_{0}\right\rangle \sum_{t} e^{-i \phi_{t}}|t\rangle$ becomes separable and $E(S, T)=0$ (stationary state), whereas if all $\left|S_{t}\right\rangle$ are orthogonal (i.e. fully distinguishable), $|\Psi\rangle$ becomes maximally entangled, with (1) already the Schmidt decomposition and $E(S, T)=\log _{2} N$ maximum. Thus, $2^{E(S, T)}$ measures the actual system evolution time, in the sense of counting the number of effective equally probable orthogonal states the system visits at orthogonal times. For constant $U_{t, t-1}$ (Eq. (6) $), E(S, T)$ is just a measure of the energy spread $(\bmod 2 \pi)$ of the initial state, as $\lambda_{k}={ }_{S}\left\langle k \mid S_{0}\right\rangle$. A similar interpretation holds for the general case in terms of the effective $H_{S}$ defined by (12).

On the other hand, the entropy determined by the conjugate distribution $\left|\Lambda_{\xi}\right|^{2}$,

$$
\tilde{E}(S, T)=-\sum_{\xi}\left|\Lambda_{\xi}\right|^{2} \log _{2}\left|\Lambda_{\xi}\right|^{2}
$$

measures the spread of $|\Psi\rangle$ over maximally entangled evolutions, or equivalently, the spread of system states $|\xi\rangle$ for a given clock state $|\tau\rangle$ (or viceversa), and is a measure of time uncertainty. It vanishes when $|\Psi\rangle$ is maximally entangled $\left(\Lambda_{\xi}=\delta_{\xi, 0}\right.$ if $\left.\lambda_{k}=\frac{1}{\sqrt{N}} \forall k\right)$, in which case there is complete synchronization between the special system and clock basis states $\left(|\Psi\rangle=\frac{1}{\sqrt{N}} \sum_{\tau}|\tau\rangle|\tau\rangle\right)$, and becomes maximum for a product state $\left(\Lambda_{\xi}=\frac{1}{\sqrt{N}}\right.$ $\forall \xi$ if $\left.\lambda_{k}=\delta_{k, 0}\right)$, in which case system and clock states are completely uncorrelated, as seen from (16). These two entropies satisfy the entropic uncertainty relation 7 (see also 11, 30 32)

$$
E(S, T)+\tilde{E}(S, T) \geq \log _{2} N
$$

which is saturated in the previous limits.

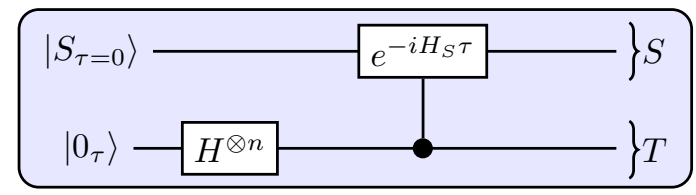

FIG. 1. Schematic circuit representing the generation of the history state (14) in the special time basis, where the system evolves according to a constant Hamiltonian $H_{S}$. Here $H^{\otimes n}$ denotes the Hadamard operator over $n$ qubits, with $2^{n}=N$. 


\section{B. The case of a complete set of evolution operators}

While for a constant Hamiltonian the system-time entanglement (19) clearly depends on the seed state $\left|S_{0}\right\rangle$, such dependence becomes softened in the more general case where the operators $U_{t, t-1}$ depend on $t$ and do not commute among themselves, i.e. when the 'Hamiltonian' $H_{t} \propto \ln U_{t, t-1}$ is time-dependent and $\left[H_{t}, H_{t^{\prime}}\right] \neq 0$ for some pairs $t \neq t^{\prime}$. If they have no common eigenstate, $|\Psi\rangle$ will be entangled for any $\left|S_{0}\right\rangle$. The extreme case is that where the $U_{t}$ 's of (4) form a complete set of orthogonal unitaries on $S$, such that

$$
\operatorname{Tr}\left[U_{t}^{\dagger} U_{t^{\prime}}\right]=d_{S} \delta_{t t^{\prime}}, \quad t, t^{\prime}=0, \ldots, d_{S}^{2}-1,
$$

implying $N=d_{S}^{2}$. In this case the history state (1) becomes maximally entangled for any initial state $\left|S_{0}\right\rangle$ :

$$
E(S, T)=\log _{2} d_{S},
$$

such that $|\Psi\rangle=\frac{1}{\sqrt{d_{S}}} \sum_{k}|k\rangle_{S}|-k\rangle_{T} \forall\left|S_{0}\right\rangle$.

Proof: We may view Eq. (22) as the scalar product between column vectors $\frac{1}{\sqrt{d_{S}}} \boldsymbol{U}_{t}$ of a $d_{S}^{2} \times d_{S}^{2}$ unitary matrix $\boldsymbol{U}$ of elements $\boldsymbol{U}_{i j, t}=\frac{1}{\sqrt{d_{S}}}\left\langle i\left|U_{t}\right| j\right\rangle$, with $\{|i\rangle\}$ any orthonormal basis of $S$, such that (22) is equivalent to $\boldsymbol{U}^{\dagger} \boldsymbol{U}=\mathbb{1}_{d_{S}^{2}}$. This matrix then satisfies as well $\boldsymbol{U} \boldsymbol{U}^{\dagger}=\mathbb{1}_{d_{S}^{2}}$, i.e. $\sum_{t}\left\langle i\left|U_{t}\right| j\right\rangle\left\langle l\left|U_{t}^{\dagger}\right| k\right\rangle=d_{S} \delta_{i k} \delta_{j l}$, which implies $\sum_{t} U_{t}|j\rangle\langle l| U_{t}^{\dagger}=d_{S} \delta_{j l} \mathbb{1}_{S}$ and hence

$$
\sum_{t} U_{t}\left|S_{0}\right\rangle\left\langle S_{0}^{\prime}\right| U_{t}^{\dagger}=d_{S}\left\langle S_{0}^{\prime} \mid S_{0}\right\rangle \mathbb{1}_{S},
$$

for any two states $\left|S_{0}\right\rangle,\left|S_{0}^{\prime}\right\rangle$ of $S$. In particular, for $\left|S_{0}\right\rangle=$ $\left|S_{0}^{\prime}\right\rangle$, Eq. 24 implies a maximally mixed reduced state $\rho_{S}=\operatorname{Tr}_{T}|\Psi\rangle\langle\Psi|$ for any seed state $\left|S_{0}\right\rangle$ :

$$
\rho_{S}=\frac{1}{d_{S}^{2}} \sum_{t} U_{t}\left|S_{0}\right\rangle\left\langle S_{0}\right| U_{t}^{\dagger}=\frac{1}{d_{S}} \mathbb{1}_{S} .
$$

Eq. (25) then leads to Eq. (23).

Therefore, a complete orthogonal set of $U_{t}$ 's ensures that the system will visit $d_{S}$ orthogonal states irrespective of the initial state $\left|S_{0}\right\rangle$. The Schmidt decomposition (11) will then select a subspace of $\mathcal{H}_{T}$ of dimension $d_{S}$ connected with $S$ through $|\Psi\rangle$. Due to the $d_{S}$-fold degeneracy $\lambda_{k}=\frac{1}{\sqrt{d_{S}}} \forall k$, any orthogonal basis $\left\{|k\rangle_{T}\right\}$ of this subspace can be used in (11), with all states $|k\rangle_{S}=\sqrt{d_{S}} T\langle-k \mid \Psi\rangle$ directly orthogonal.

A convenient choice of complete orthogonal set is provided by the Weyl operators 33 35

$$
U_{t} \equiv U_{p q}=\exp \left[i 2 \pi p Q / d_{S}\right] \exp \left[-i 2 \pi q P / d_{S}\right],
$$

where $p, q=0, \ldots, d_{S}-1, t=q d_{S}+p, Q|q\rangle=q|q\rangle$, $P|p\rangle=p|p\rangle$ and $\{|q\rangle\},\{|p\rangle\}$ are orthogonal bases of $S$ related through a DFT: $|p\rangle=\frac{1}{\sqrt{d_{S}}} \sum_{q} e^{i 2 \pi p q / d_{S}}|q\rangle$. They satisfy, for any eigenstate $\left|q_{0}\right\rangle$ of $Q$,

$$
U_{p q}\left|q_{0}\right\rangle=e^{i 2 \pi p\left(q_{0}+q\right) / d_{S}}\left|q_{0}+q\right\rangle
$$

which implies Eq. (22), i.e. $\operatorname{Tr} U_{p^{\prime} q^{\prime}}^{\dagger} U_{p q}=d_{S} \delta_{q^{\prime} q} \delta_{p^{\prime} p}$.

The discrete evolution under these operators can then be achieved by application of just two different unitaries $U_{t, t-1}$ to the preceding state (here $m \geq 1$, integer):

$$
U_{t, t-1}=\left\{\begin{array}{ll}
e^{i 2 \pi Q / d_{S}} & t \neq m d_{S} \\
e^{-i 2 \pi P / d_{S}} e^{i 2 \pi Q / d_{S}} & t=m d_{S}
\end{array} .\right.
$$

For instance, if $S$ is a qubit $\left(d_{S}=2\right)$ we may take $Q=\left(\mathbb{1}-\sigma_{z}\right) / 2, P=\left(\mathbb{1}-\sigma_{x}\right) / 2$, with $e^{i 2 \pi Q / d_{S}}=\sigma_{z}$, $e^{-i 2 \pi P / d_{S}}=\sigma_{x}$. Hence, $|\Psi\rangle=\frac{1}{2}\left[\left|S_{0}\right\rangle|0\rangle+\sigma_{z}\left|S_{0}\right\rangle|1\rangle+\right.$ $\left.\sigma_{x}\left|S_{0}\right\rangle|2\rangle+i \sigma_{y}\left|S_{0}\right\rangle|3\rangle\right]$ is maximally entangled $\forall\left|S_{0}\right\rangle$ $(E(S, T)=1)$, with $\left|S_{1}\right\rangle=\sigma_{z}\left|S_{0}\right\rangle,\left|S_{2}\right\rangle=\sigma_{x}\left|S_{0}\right\rangle=$ $-i \sigma_{y}\left|S_{1}\right\rangle,\left|S_{3}\right\rangle=i \sigma_{y}\left|S_{0}\right\rangle=\sigma_{z}\left|S_{2}\right\rangle$ and $\left|S_{0}\right\rangle=-i \sigma_{y}\left|S_{3}\right\rangle$.

In the general case, it is here natural to view system $T$ as formed by two clocks with identical Hilbert space dimension $d_{S}$, which govern time-independent Hamiltonians $H_{1}=-2 \pi Q / d_{S}$ and $H_{2}=2 \pi P / d_{S}$ associated with conjugate operators $Q, P$ on $S$. Then we may write the history state (1) for the operators (26) as

$$
|\Psi\rangle=\frac{1}{d_{S}^{2}} \sum_{p, q} U_{p q}\left|S_{0}\right\rangle|p\rangle_{T_{1}}|q\rangle_{T_{2}},
$$

which represents a history state of history states. It can then be implemented with the circuit of Fig. 2

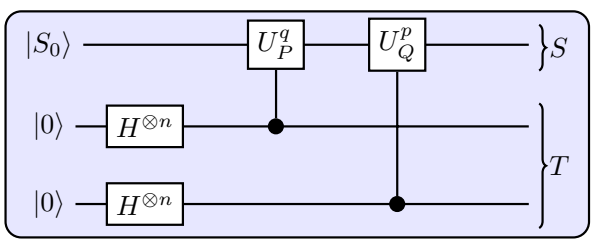

FIG. 2. Schematic circuit representing the generation of a maximally entangled history state $|\Psi\rangle$, for any initial system state $\left|S_{0}\right\rangle$. Here $U_{P}=e^{-i 2 \pi P / d_{S}}, U_{Q}=e^{i 2 \pi Q / d_{S}}$, with $P, Q$ conjugate operators on $S$ and $2^{n}=d_{S}$.

\section{The quadratic $S-T$ entanglement entropy: Analytic evaluation and bounds}

The analytic evaluation of the entropy (19) in the general case requires the determination of the singular values $\lambda_{k}$, i.e., the eigenvalues $\lambda_{k}^{2}$ of $\rho_{S}$ or $\rho_{T}$, which is difficult in most cases. It is then convenient to use the quadratic (also called linear) entropy $S_{2}(\rho)=2 \operatorname{Tr}[\rho(\mathbb{1}-\rho)]=$ $2\left(1-\operatorname{Tr} \rho^{2}\right)$, which does not require explicit knowledge of the eigenvalues and is a linear function of the purity $\operatorname{Tr} \rho^{2}$. Like $S(\rho)$, it vanishes iff $\rho$ is pure and is maximum iff $\rho$ is maximally mixed (with $S_{2}(\rho)=1$ for a maximally mixed single qubit state), satisfying the majorization relation $S_{2}\left(\rho^{\prime}\right) \geq S_{2}(\rho)$ if $\rho^{\prime} \prec \rho$ 36, 37. The associated $S-T$ entanglement entropy is

$$
\begin{aligned}
E_{2}(S, T) & =S_{2}\left(\rho_{S}\right)=S_{2}\left(\rho_{T}\right)=2\left(1-\sum_{k} \lambda_{k}^{4}\right) \\
& =2\left(1-\frac{1}{N^{2}} \sum_{t, t^{\prime}}\left|\left\langle S_{t} \mid S_{t^{\prime}}\right\rangle\right|^{2}\right),
\end{aligned}
$$


and can be determined just from the overlaps between the evolved states. For the complete orthogonal set (22), it is easily verified that $\sum_{t, t^{\prime}}\left|\left\langle S_{t} \mid S_{t^{\prime}}\right\rangle\right|^{2}=d_{S}^{3}$, so that $E_{2}(S, T)=2\left(1-\frac{1}{d_{S}}\right)$ becomes maximum.

The overlaps $\left\langle S_{t} \mid S_{t^{\prime}}\right\rangle$ are also experimentally accessible through a measurement at the clock $T$ of the nondiagonal operators $\left|t^{\prime}\right\rangle\langle t|\left(t \neq t^{\prime}\right)$ :

$$
\frac{1}{N}\left\langle S_{t^{\prime}} \mid S_{t}\right\rangle=\left\langle\Psi\left|\mathbb{1}_{S} \otimes\right| t^{\prime}\right\rangle\langle t|| \Psi\rangle=\left\langle\sigma_{t^{\prime} t}^{x}\right\rangle+i\left\langle\sigma_{t^{\prime} t}^{y}\right\rangle,
$$

where $\sigma_{t^{\prime} t}^{x}=\left|t^{\prime}\right\rangle\langle t|+| t\rangle\left\langle t^{\prime}\right|, \sigma_{t^{\prime} t}^{y}=\left(\left|t^{\prime}\right\rangle\langle t|-| t\rangle\left\langle t^{\prime}\right|\right) / i$ are hermitian Pauli operators for the pair $t \neq t^{\prime}$.

Let us now consider the evolution for a general constant Hamiltonian $H$ of arbitrary spectrum for system $S$, such that $U_{t}=e^{-i H t} \forall t$. In contrast with (19), Eq. (31) can in this case be explicitly evaluated. Writing

$$
\left|S_{0}\right\rangle=\sum_{k} c_{k}\left|E_{k}\right\rangle, \quad H\left|E_{k}\right\rangle=E_{k}\left|E_{k}\right\rangle
$$

with $E_{k} \neq E_{k^{\prime}}$ if $k \neq k^{\prime}$ (in case of degenerate states $\left|k_{l}\right\rangle, c_{k}\left|E_{k}\right\rangle=\sum_{l} c_{k l}\left|k_{l}\right\rangle$, with $\left|c_{k}\right|^{2}=\sum_{l}\left|c_{k l}\right|^{2}$ ), then $\left|S_{t}\right\rangle=\sum_{k} e^{-i E_{k} t} c_{k}\left|E_{k}\right\rangle$ and Eq. (31) becomes, for equally spaced times $t=t_{f} \frac{j}{N-1}, j=0, \ldots, N-1$,

$$
\begin{aligned}
E_{2}(S, T) & =2\left(1-\left.\left.\frac{1}{N^{2}} \sum_{t, t^{\prime}}\left|\sum_{k}\right| c_{k}\right|^{2} e^{-i E_{k}\left(t-t^{\prime}\right)}\right|^{2}\right) \\
& =2 \sum_{k \neq k^{\prime}}\left|c_{k} c_{k^{\prime}}\right|^{2}\left[1-\frac{\sin ^{2} \frac{\left(E_{k}-E_{k^{\prime}}\right) t_{f} N}{2(N-1)}}{N^{2} \sin ^{2} \frac{\left(E_{k}-E_{k^{\prime}}\right) t_{f}}{2(N-1)}}\right] .
\end{aligned}
$$

The exact result for a continuous evolution can also be obtained from (35), by taking the limit $N \rightarrow \infty$ :

$$
E_{2}(S, T) \underset{N \rightarrow \infty}{\rightarrow} 2 \sum_{k \neq k^{\prime}}\left|c_{k} c_{k^{\prime}}\right|^{2}\left[1-\frac{\sin ^{2}\left(\frac{\left(E_{k}-E_{k^{\prime}}\right) t_{f}}{2}\right)}{\left(\frac{\left(E_{k}-E_{k^{\prime}}\right) t_{f}}{2}\right)^{2}}\right]
$$

Eq. (36) provides a good approximation to (35) if $\frac{\left|E_{k}-E_{k^{\prime}}\right| t_{f}}{N-1} \ll 1 \forall k \neq k^{\prime}$ with finite weight $\left|c_{k} c_{k^{\prime}}\right|^{2}>0$.

Eqs. (35) - 36) are essentially measures of the spread of $\left|S_{0}\right\rangle$ over distinct energy eigenstates. For small $t_{f}$ such that $\left|E_{k}-E_{k^{\prime}}\right| t_{f} \ll 1 \forall k, k^{\prime}$, a second order expansion shows they are proportional to the energy fluctuation in $\left|S_{0}\right\rangle:\left|\left\langle S_{t} \mid S_{t^{\prime}}\right\rangle\right|^{2} \approx 1-\left\langle(\Delta H)^{2}\right\rangle\left(t-t^{\prime}\right)^{2}$, with $\Delta H=$ $H-\langle H\rangle$ and $\langle O\rangle=\left\langle S_{0}|O| S_{0}\right\rangle$, implying

$$
E_{2}(S, T) \approx \frac{N+1}{3(N-1)}\left\langle(\Delta H)^{2}\right\rangle t_{f}^{2} \underset{N \rightarrow \infty}{\rightarrow} \frac{1}{3}\left\langle(\Delta H)^{2}\right\rangle t_{f}^{2} .
$$

It then becomes proportional to the square of the speed $\sqrt{\left\langle(\Delta H)^{2}\right\rangle}$ of the continuous quantum evolution according to the Fubini-Study metric 19, 38.

It is also apparent from (35) that $E_{2}(S, T)$ is upper bounded by the quadratic entropy of the energy distribution $\left|c_{k}\right|^{2}$ :

$$
E_{2}(S, T) \leq 2 \sum_{k \neq k^{\prime}}\left|c_{k} c_{k^{\prime}}\right|^{2}=2\left(1-\sum_{k}\left|c_{k}\right|^{4}\right) .
$$

The maximum for a fixed distribution $\left|c_{k}\right|^{2}$ is reached for an equally spaced spectrum of the form

$$
E_{k}=\frac{N-1}{t_{f}} \frac{2 \pi k}{N}+C
$$

with $k$ integer $\in[0, N-1]$, since in this case the bracket in (35) takes its maximum value $1 \forall k \neq k^{\prime}$.

The spectrum (39) is just Eq. (12) for the scaled Hamiltonian $H_{S}=\frac{t_{f}}{N-1}(H-C)$ (for which $\left.t=0, \ldots, N-1\right)$, so that the energy states $\left|E_{k}\right\rangle$ become the Schmidt states $|k\rangle_{S}$ of (11) and $\left|c_{k}\right|$ the Schmidt coefficients $\lambda_{k}$. For other spectra, the states $|\tilde{k}\rangle_{T}=\frac{1}{\sqrt{N}} \sum_{t} e^{-i E_{k} t}|t\rangle$ in

$$
|\Psi\rangle=\frac{1}{\sqrt{N}} \sum_{k, t} c_{k} e^{-i E_{k} t}\left|E_{k}\right\rangle|t\rangle=\sum_{k} c_{k}\left|E_{k}\right\rangle|\tilde{k}\rangle_{T},
$$

are not necessarily all orthogonal, so that $E(S, T)$ will become normally smaller [7]. Nonetheless, for large $N$ and not too small $t_{f}$, the states $|\tilde{k}\rangle_{T}$ will typically be almost orthogonal, so that the deviation from the upper bound (38) will not be large, becoming significant only in the presence of quasidegeneracies in the spectrum: The bracket in (36) vanishes just for $E_{k} \rightarrow E_{k^{\prime}}$, becoming close to 1 for $\left|E_{k}-E_{k^{\prime}}\right| t_{f} / 2>\pi$, while that in (35), which is a periodic function of $E_{k}-E_{k^{\prime}}$ with period $\Delta_{N}=$ $2 \pi \frac{N-1}{t_{f}}$, vanishes for $E_{k} \rightarrow E_{k^{\prime}}+m \Delta_{N}, m=0$ or integer, becoming close to 1 whenever $\left|E_{k}-E_{k^{\prime}}-m \Delta_{N}\right| t_{f} / 2>\pi$.

On the other hand, Eq. (36) also admits a lower bound for fixed initial and final states $\left|S_{0}\right\rangle$ and $\left|S_{t_{f}}\right\rangle=$ $\sum_{k} c_{k} e^{-i E_{k} t_{f}}\left|E_{k}\right\rangle$, reached when the evolution (over $N$ equally spaced times $t=t_{f} \frac{j}{N-1}$ under a constant $H$ ) remains in the subspace spanned by $\left|S_{0}\right\rangle$ and $\left|S_{t_{f}}\right\rangle$ :

$$
E_{2}(S, T) \geq E_{2}^{\min }(S, T)=1-\frac{\sin ^{2} \frac{N \phi}{N-1}}{N^{2} \sin ^{2} \frac{\phi}{N-1}},
$$

where $\phi \in[0, \pi / 2]$ is determined by the overlap between the initial and final states:

$$
\cos \phi=\left|\left\langle S_{0} \mid S_{t_{f}}\right\rangle\right|=\left.\left|\sum_{k}\right| c_{k}\right|^{2} e^{-i E_{k} t_{f}} \mid .
$$

Writing the final state as

$$
\left|S_{t_{f}}\right\rangle=e^{-i \gamma}\left(\cos \phi\left|S_{0}\right\rangle+\sin \phi\left|S_{0}^{\perp}\right\rangle\right),
$$

where $\left\langle S_{0}^{\perp} \mid S_{0}\right\rangle=0, E_{2}^{\min }(S, T)$ is the result of Eq. (35. for an evolution under a two level Hamiltonian

$$
H^{\mathrm{min}}=\frac{\phi}{t_{f}} \sigma_{y}+\frac{\gamma}{t_{f}}, \quad \sigma_{y}=-i\left(\left|S_{0}\right\rangle\left\langle S_{0}^{\perp}|-| S_{0}^{\perp}\right\rangle\left\langle S_{0}\right|\right),
$$

such that

$$
\begin{aligned}
\left|S_{t}^{\min }\right\rangle & \equiv \exp \left[-i H^{\min } t\right]\left|S_{0}\right\rangle \\
& =e^{-i \gamma t / t_{f}}\left(\cos \frac{\phi t}{t_{f}}\left|S_{0}\right\rangle+\sin \frac{\phi t}{t_{f}}\left|S_{0}^{\perp}\right\rangle\right),
\end{aligned}
$$

with $\left|S_{t_{f}}^{\min }\right\rangle=\left|S_{t_{f}}\right\rangle$. 
The demonstration of (41) is given in the appendix, but the result is physically clear: The $S-T$ entanglement is a measure of the distinguishability between the evolved states, and the minimum value is then obtained for an evolution within the subspace containing the initial and final states, where all intermediate states will be closer than in a general evolution. Such evolution, Eq. (45), proceeds precisely along the geodesic determined by the Fubini-Study metric 19, 38, saturating the MandelstamTamm bound [39] $\Delta t \Delta E \geq \cos ^{-1}\left(\left|\left\langle S_{0} \mid S_{t_{f}}\right\rangle\right|\right)=\phi(\Delta t=$ $\left.t_{f}, \Delta E=\sqrt{\left\langle\left(\Delta H^{\min }\right)^{2}\right\rangle}=\phi / t_{f}\right)$.

As check, for small $t_{f}$ such that $\left|E_{k}-E_{k^{\prime}}\right| t_{f} \ll 1 \forall k \neq$ $k^{\prime}$, a fourth order expansion of (35) and (41) leads to

$E_{2}(S, T)-E_{2}^{\min }(S, T) \approx \kappa\left[\left\langle(\Delta H)^{4}\right\rangle-\left\langle(\Delta H)^{2}\right\rangle^{2}\right] t_{f}^{4} \geq 0$,

where $\kappa=\frac{(N+1)(N-2)(N-4 / 3)}{60(N-1)^{3}}>0 \forall N>2$. Hence, the difference (46) is verified to be non-negative and of fourth order in $t_{f}$, being proportional to the fluctuation of $(\Delta H)^{2}$. The latter vanishes just for the geodesic evolution, where $\Delta H=\Delta H^{\min }=\frac{\phi}{t_{f}} \sigma_{y}$ and hence $\left\langle\left(\Delta H^{\mathrm{min}}\right)^{4}\right\rangle=\left\langle\left(\Delta H^{\mathrm{min}}\right)^{2}\right\rangle^{2}=\phi^{4} / t_{f}^{4}$, implying $E_{2}(S, T)=E_{2}^{\min }(S, T)$. Such fluctuation represents a curvature coefficient which measures the deviation from the geodesic [38, 40].

For $\phi \in[0, \pi / 2]$, the bound (41) is, of course, an increasing function of $\phi$ for $N \geq 2$, i.e. of the Wootters distance 41] $s\left(\left|S_{0}\right\rangle,\left|S_{t_{f}}\right\rangle\right)=2 \arccos \left(\left|\left\langle S_{0} \mid S_{t_{f}}\right\rangle\right|\right)=2 \phi$, and hence a decreasing function of the overlap $\left|\left\langle S_{t_{f}} \mid S_{0}\right\rangle\right|$. It is also a decreasing function of $N \geq 2$ for $\phi \in(0, \pi / 2]$. The minimum value is thus achieved in the continuous limit $N \rightarrow \infty$, where $E_{2}^{\min }(S, T) \rightarrow 1-\left(\sin ^{2} \phi\right) / \phi^{2}$. Then, we may also write, for any $N \geq 2$,

$$
E_{2}(S, T) \geq 1-\frac{\sin ^{2} \phi}{\phi^{2}} \text {. }
$$

\section{ENTANGLEMENT AND HISTORY STATES OF EVOLUTION OPERATORS}

We now examine the application of the previous formalism to the evolution operators themselves. The aim is to link properties of previous history states with those of the operators that generate it. For this purpose the pure state representation of operators 24 28] provides a convenient approach, enabling a direct derivation of their entanglement properties 20 23.

\section{A. Entanglement of operators and pure state representation}

We first briefly review the concept of operator entanglement and its pure state representation. Any operator $\mathcal{W}$ for a bipartite system $\mathrm{A}+\mathrm{B}$ can be expanded as

$$
\mathcal{W}=\sum_{i, j} M_{i j} C_{i} \otimes D_{j}
$$

where $C_{i}$ and $D_{j}$ are orthogonal operators for $\mathrm{A}$ and $\mathrm{B}$ respectively, satisfying

$$
\operatorname{Tr} C_{i}^{\dagger} C_{j}=\delta_{i j} d_{A}, \quad \operatorname{Tr} D_{i}^{\dagger} D_{j}=\delta_{i j} d_{B} .
$$

Hence, $M_{i j}=\frac{1}{d_{A} d_{B}} \operatorname{Tr}\left[C_{i}^{\dagger} \otimes D_{j}^{\dagger} \mathcal{W}\right]$. We can use, for instance, the Weyl operators (26) for the sets $\left\{C_{i}\right\},\left\{D_{i}\right\}$.

Eqs. [49) imply $\operatorname{Tr}\left[\mathcal{W}^{\dagger} \mathcal{W}\right]=d_{A} d_{B} \operatorname{Tr}\left[M^{\dagger} M\right]$. If $\mathcal{W}$ is unitary, then $\operatorname{Tr}\left[M^{\dagger} M\right]=1$, entailing that the numbers $\left\{\left|M_{i j}\right|^{2}\right\}$ are in this case standard probabilities. By means of the singular value decomposition, we can write the $d_{A}^{2} \times d_{B}^{2}$ matrix $M$ as $M=U D V^{\dagger}$, where $U$ and $V$ are unitary matrices and $D$ a diagonal matrix with nonnegative entries $\lambda_{k}^{\mathcal{W}}$ satisfying $\sum_{k}\left(\lambda_{k}^{\mathcal{W}}\right)^{2}=\operatorname{Tr} M^{\dagger} M=1$. We can then rewrite $\mathcal{W}$ in the $\mathrm{Schmidt}$ form

$$
\mathcal{W}=\sum_{k} \lambda_{k}^{\mathcal{W}} A_{k} \otimes B_{k}
$$

where $A_{k} \equiv \sum_{i} U_{i k} C_{i}$ and $B_{k} \equiv \sum_{j} V_{j k}^{*} D_{j}$, are again orthogonal operator bases for $A$ and $B$ satisfying $\operatorname{Tr} A_{k}^{\dagger} A_{l}=$ $d_{A} \delta_{k l}, \operatorname{Tr} B_{k}^{\dagger} B_{l}=d_{B} \delta_{k l}$. The von Neumann entanglement entropy of $\mathcal{W}$ can then be defined as

$$
E(\mathcal{W})=-\sum_{k}\left(\lambda_{k}^{\mathcal{W}}\right)^{2} \log _{2}\left(\lambda_{k}^{\mathcal{W}}\right)^{2}
$$

Similarly, $E_{2}(\mathcal{W})=2 \sum_{k}\left(1-\left(\lambda_{k}^{\mathcal{W}}\right)^{4}\right)$. These entropies vanish when $\mathcal{W}$ is a product of local unitaries, and are maximum when $\mathcal{W}$ is a uniform sum of $d^{2}$ products $A_{k} \otimes$ $B_{k}$, with $d=\operatorname{Min}\left[d_{A}, d_{B}\right]$.

The previous analogy between operators and states can be manifestly described through the Choi isomorphism 24 28. Any operator $O$ in a system with Hilbert space $\mathcal{H}$ of dimension $d$ can be associated with a pure state $|O\rangle \in \mathcal{H} \otimes \mathcal{H}$, given by

$|O\rangle=(O \otimes \mathbb{1})|\mathbb{1}\rangle=\frac{1}{\sqrt{d}} \sum_{q}(O|q\rangle)|q\rangle=\frac{1}{\sqrt{d}} \sum_{q, q^{\prime}}\left\langle q^{\prime}|O| q\right\rangle\left|q^{\prime}\right\rangle|q\rangle$

where $|\mathbb{1}\rangle=\frac{1}{\sqrt{d}} \sum_{q}|q\rangle|q\rangle$ is a maximally entangled state in $\mathcal{H} \otimes \mathcal{H}$ and $\{|q\rangle\}$ an orthonormal set. In this way,

$$
\left\langle O \mid O^{\prime}\right\rangle=\frac{1}{d} \operatorname{Tr}\left[\mathrm{O}^{\dagger} \mathrm{O}^{\prime}\right]
$$

Therefore, orthogonal operators satisfying $\operatorname{Tr}\left[O_{i}^{\dagger} O_{j}\right]=$ $d \delta_{i j}$ correspond to orthonormal states $\left\langle O_{i} \mid O_{j}\right\rangle=\delta_{i j}$. And unitary operators $U$ to normalized states $|U\rangle$.

The operator (48) can then be associated with the pure state (note that $\left|\mathbb{1}_{A B}\right\rangle=\left|\mathbb{1}_{A}\right\rangle\left|\mathbb{1}_{B}\right\rangle$ )

$$
|\mathcal{W}\rangle=\left(\mathcal{W} \otimes \mathbb{1}_{A^{\prime} B^{\prime}}\right)\left|\mathbb{1}_{A}\right\rangle\left|\mathbb{1}_{B}\right\rangle=\sum_{i j} M_{i j}\left|C_{i}\right\rangle\left|D_{j}\right\rangle
$$

where $\left|C_{i}\right\rangle=\left(C_{i} \otimes \mathbb{1}_{A^{\prime}}\right)\left|\mathbb{1}_{A}\right\rangle,\left|D_{j}\right\rangle=\left(D_{j} \otimes \mathbb{1}_{B^{\prime}}\right)\left|\mathbb{1}_{B}\right\rangle$ form orthogonal sets: $\left\langle C_{k} \mid C_{i}\right\rangle=\delta_{k i},\left\langle D_{k} \mid D_{j}\right\rangle=\delta_{k j}$. Thus, $M_{i j}=\left\langle C_{i}, D_{j} \mid \mathcal{W}\right\rangle$, with $\langle\mathcal{W} \mid \mathcal{W}\rangle=\operatorname{Tr}\left[M^{\dagger} M\right]$. 
The state representation of the Schmidt form (50) acquires then the standard appearance

$$
|\mathcal{W}\rangle=\sum_{k} \lambda_{k}^{\mathcal{W}}\left|A_{k}\right\rangle\left|B_{k}\right\rangle
$$

with $\left\langle A_{k} \mid A_{l}\right\rangle=\delta_{k l}=\left\langle B_{k} \mid B_{l}\right\rangle$, and the entanglement entropy (51) of a unitary $\mathcal{W}$ can be also expressed as

$$
E(\mathcal{W})=S\left(\rho_{A}^{\mathcal{W}}\right)=S\left(\rho_{B}^{\mathcal{W}}\right), \quad \rho_{A(B)}^{\mathcal{W}}=\operatorname{Tr}_{B(A)}|\mathcal{W}\rangle\langle\mathcal{W}|
$$

with $S(\rho)=-\operatorname{Tr} \rho \log _{2} \rho$. Similarly, $E_{2}(\mathcal{W})=S_{2}\left(\rho_{A}^{\mathcal{W}}\right)=$ $S_{2}\left(\rho_{B}^{\mathcal{W}}\right)$, with $S_{2}(\rho)=2\left(1-\operatorname{Tr} \rho^{2}\right)$.

\section{B. Generating operators and operator history states}

The history state (10) can be generated from an initial product state $\left|S_{0}\right\rangle|0\rangle$ as

$$
|\Psi\rangle=\mathcal{W}\left(I \otimes H^{\otimes n}\right)\left|S_{0}\right\rangle|0\rangle,
$$

where $H^{\otimes n}$ denotes the Hadamard operator acting on the clock $\left(H^{\otimes n}|0\rangle=\frac{1}{\sqrt{N}} \sum_{t=0}^{N-1}|t\rangle\right.$, with $\left.N=2^{n}\right)$ and

$$
\mathcal{W}=\sum_{t} U_{t} \otimes|t\rangle\langle t|
$$

the control- $U_{t}$ operator. By expanding $U_{t}$ in an orthogonal basis of operators $C_{i}$, we have

$$
\mathcal{W}=\sum_{t, i} M_{t i} C_{i} \otimes|t\rangle\langle t|, \quad M_{t i}=\frac{1}{d_{S}} \operatorname{Tr} C_{i}^{\dagger} U_{t}
$$

where the coefficients $M_{t j}$ satisfy $\sum_{j}\left|M_{t j}\right|^{2}=$ $\frac{1}{d_{S}} \operatorname{Tr} U_{t}^{\dagger} U_{t}=1$, and are hence standard probabilities at fixed $t$. Since the projectors $|t\rangle\langle t|$ are also orthogonal and have unit trace, the Schmidt coefficient $\lambda_{k}^{\mathcal{W}}$ of (50) are here just the singular values of the matrix $M / \sqrt{N}$. The ensuing entanglement entropy (51) is the same as that of $\mathcal{W}\left(I \otimes H^{\otimes n}\right)$, as they differ just by a local unitary.

The pure state (54) associated with the operator (58) is itself an operator history state:

$$
|\mathcal{W}\rangle=\frac{1}{\sqrt{N}} \sum_{t}\left|U_{t}\right\rangle\left|T_{t}\right\rangle
$$

where $\left|U_{t}\right\rangle=\left(U_{t} \otimes \mathbb{1}_{S^{\prime}}\right)\left|\mathbb{1}_{S}\right\rangle=\frac{1}{\sqrt{d_{S}}} \sum_{q} U_{t}|q\rangle|q\rangle$ and $\left|T_{t}\right\rangle=\left(T_{t} \otimes \mathbb{1}_{T^{\prime}}\right)\left|\mathbb{1}_{T}\right\rangle=|t t\rangle$, with $T_{t}=\sqrt{N}|t\rangle\langle t|$ and $\left\langle T_{t} \mid T_{t^{\prime}}\right\rangle=\delta_{t t^{\prime}}$. Writing $|t t\rangle$ simply as $|t\rangle$, Eq. (60) is the standard history state (10) for a maximally entangled initial state $\left|\mathbb{1}_{S}\right\rangle=\frac{1}{\sqrt{d_{s}}} \sum_{q}|q\rangle|q\rangle$ of a bipartite system under a local evolution $U_{t} \otimes \mathbb{1}_{S^{\prime}}$, so that it can be generated with the circuit depicted in Fig. 3.

The entanglement of the history state (60) is the operator entanglement (51) of $\mathcal{W}$, which is then a measure of the distinguishability of the operator states $\left|U_{t}\right\rangle$. Its

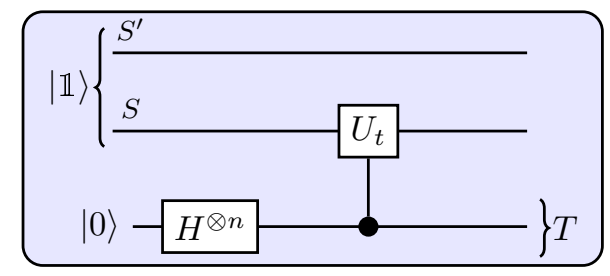

FIG. 3. (Color online) Schematic circuit representing the generation of the operator history state 60.

quadratic entanglement can be directly evaluated with Eq. (31), where now $\left\langle U_{t} \mid U_{t^{\prime}}\right\rangle=\frac{1}{d_{S}} \operatorname{Tr}\left[U_{t}^{\dagger} U_{t^{\prime}}\right]$ :

$$
E_{2}(\mathcal{W})=2\left(1-\frac{1}{N^{2}} \sum_{t, t^{\prime}}\left|\left\langle U_{t} \mid U_{t^{\prime}}\right\rangle\right|^{2}\right) .
$$

It is now immediate to see that if $N=d_{S}^{2}$ and the operators $\left\{U_{t}\right\}$ form a complete orthogonal set (Eq. (22)), the operator history state (60) is maximally entangled:

$$
E(\mathcal{W})=\log _{2} d_{S}^{2}=2 \log _{2} d_{S},
$$

while $E_{2}(\mathcal{W})=2\left(1-\frac{1}{d_{S}^{2}}\right)$, since all states $\left|U_{t}\right\rangle$ become orthogonal: $\left\langle U_{t} \mid U_{t^{\prime}}\right\rangle=\delta_{t t^{\prime}}$. The form (60) is then already the Schmidt decomposition of $|\mathcal{W}\rangle$. Since in this case the original history state (1) has maximum entanglement $E(S, T)=\log _{2} d_{S}$ for any initial state $\left|S_{0}\right\rangle$, this result indicates a close relation between the entangling power of $\mathcal{W}$ and its operator entanglement, which will be discussed below. It is also apparent that if the $d_{S}^{2}$ operators $U_{t}$ are not all orthogonal, then $E(\mathcal{U})<2 \log _{2} d_{S}$.

For a smaller number $N<d_{S}^{2}$ of times, $E(\mathcal{W})$ will be maximum if all $N$ states $\left|U_{t}\right\rangle$ are orthogonal. In the case of a constant Hamiltonian with energies $E_{k}$, such that $U_{t}=e^{-i H t} \forall t$, then

$$
\left\langle U_{t} \mid U_{t^{\prime}}\right\rangle=\frac{1}{d_{S}} \sum_{k} e^{-i E_{k}\left(t-t^{\prime}\right)} .
$$

For $N=d_{S}$, an equally spaced spectrum $E_{k}=2 \pi k / N+$ $C, k=0, \ldots, N-1$, (i.e., Eq. (39) if $t \rightarrow \frac{t_{f}}{N-1} j$ ) ensures that all $\left|U_{t}\right\rangle$ are strictly orthogonal: $\left\langle U_{t} \mid U_{t^{\prime}}\right\rangle=\delta_{t t^{\prime}} \forall t, t^{\prime}$ (the ensuing operators $U_{t}$ are in fact the first $d_{S}$ operators of the Weyl set (26) ). Hence, $E(\mathcal{W})$ will reach for this spectrum the maximum value

$$
E(\mathcal{W})=\log _{2} d_{S},
$$

compatible with a fixed $H$ and $N=d_{S}$ times. The same holds for $E_{2}(\mathcal{W})$. This result correlates with the extremal properties of this spectrum discussed in $\amalg \mathrm{IC}$

On the other hand, since $U_{t, t-1}=U_{t} U_{t-1}^{\dagger}$, the operator $\mathcal{U}$ of Eq. (2) is related with $\mathcal{W}$ by

$$
\mathcal{U}=\mathcal{W}\left(I \otimes \exp \left[-i P_{T}\right]\right) \mathcal{W}^{\dagger},
$$

where $\exp \left[-i P_{T}\right]=\sum_{t}|t\rangle\langle t-1|$. The associated pure state is also a history state,

$$
|\mathcal{U}\rangle=\frac{1}{\sqrt{N}} \sum_{t}\left|U_{t, t-1}\right\rangle\left|T_{t, t-1}\right\rangle,
$$


where $\left|T_{t, t-1}\right\rangle=\sqrt{N}\left(|t\rangle\langle t-1| \otimes \mathbb{1}_{T^{\prime}}\right)\left|\mathbb{1}_{T}\right\rangle=|t, t-1\rangle$ are again orthogonal states. Its entanglement is then a measure of the distinguishability of the step evolution operator states $\left|U_{t, t-1}\right\rangle$, and depends on the order of the operators $U_{t}$, in contrast with $E(\mathcal{W})$. It vanishes in the constant case (6)-(7).

\section{Operator entanglement and entangling power}

We have seen that there is a relation between the entanglement of the operator $\mathcal{W}$ and that of the history states it generates, $|\Psi\rangle=\frac{1}{\sqrt{N}} \sum_{t} U_{t}\left|S_{0}\right\rangle|t\rangle$. We will here prove that the quadratic operator entanglement entropy $E_{2}(U, T) \equiv E_{2}(\mathcal{W})$, Eq. [61), is proportional to the entangling power of $\mathcal{W}$, defined as the average quadratic entanglement it generates when applied (as in Eq. (57)) to initial product states $\left|S_{0}\right\rangle|0\rangle$ :

$$
\left\langle E_{2}(S, T)\right\rangle=\frac{d_{S}}{d_{S}+1} E_{2}(\mathcal{W})
$$

where

$$
\left\langle E_{2}(S, T)\right\rangle=\int_{\mathcal{H}} 2\left(1-\operatorname{Tr} \rho_{S}^{2}\right) d S_{0}
$$

is the average over all initial states $\left|S_{0}\right\rangle$ of the quadratic entanglement entropy $E_{2}(S, T)$ of the history state: The integral runs over the whole set of initial states $\left|S_{0}\right\rangle$ with the Haar measure $d S_{0}$ (the only normalized unitarily invariant measure over the Hilbert space) and $\rho_{S}$ is the reduced state of $S$ in $|\Psi\rangle$.

Proof. Since $\rho_{S}=\frac{1}{N} \sum_{t} U_{t}\left|S_{0}\right\rangle\left\langle S_{0}\right| U_{t}^{\dagger}$, we obtain

$$
\left\langle\operatorname{Tr} \rho_{S}^{2}\right\rangle=\frac{1}{N^{2}} \sum_{t, t^{\prime}} \int_{\mathcal{H}}\left\langle S_{0}\left|U_{t}^{\dagger} U_{t^{\prime}}\right| S_{0}\right\rangle\left\langle S_{0}\left|U_{t^{\prime}}^{\dagger} U_{t}\right| S_{0}\right\rangle d S_{0}
$$

Here we can define $O=U_{t}^{\dagger} U_{t^{\prime}}$ and $P=U_{t^{\prime}}^{\dagger} U_{t}=O^{\dagger}$ to use the relation 42 .

$$
\int_{\mathcal{H}}\left\langle S_{0}|O| S_{0}\right\rangle\left\langle S_{0}|P| S_{0}\right\rangle d S_{0}=\frac{\operatorname{Tr}[O] \operatorname{Tr}[P]+\operatorname{Tr}[O P]}{d_{S}\left(d_{S}+1\right)} .
$$

Since in this case $O P=\mathbb{1}_{S}$, we obtain

$$
\left\langle\operatorname{Tr} \rho_{S}^{2}\right\rangle=\frac{\frac{1}{N^{2}} \sum_{t, t^{\prime}}\left|\operatorname{Tr}\left[U_{t}^{\dagger} U_{t^{\prime}}\right]\right|^{2}+d_{S}}{d_{S}\left(d_{S}+1\right)} .
$$

On the other hand, $E_{2}(\mathcal{W})=2\left(1-\operatorname{Tr} \rho_{U}^{2}\right)$, with $\rho_{U}^{2}=$ $\frac{1}{N^{2}} \sum_{t, t^{\prime}}\left|U_{t}\right\rangle\left\langle U_{t}^{\dagger} \mid U_{t^{\prime}}\right\rangle\left\langle U_{t^{\prime}}^{\dagger}\right|$. Thus,

$$
\operatorname{Tr} \rho_{U}^{2}=\frac{1}{N^{2}} \sum_{t, t^{\prime}}\left|\left\langle U_{t}^{\dagger} \mid U_{t^{\prime}}\right\rangle\right|^{2}=\frac{1}{\left(d_{S} N\right)^{2}} \sum_{t, t^{\prime}}\left|\operatorname{Tr}\left[U_{t}^{\dagger} U_{t^{\prime}}\right]\right|^{2} .
$$

Replacing (72) in (71) leads to $\left\langle\operatorname{Tr} \rho_{S}^{2}\right\rangle=\frac{d_{S} \operatorname{Tr}\left(\rho_{U}^{2}\right)+1}{d_{S}+1}$ and hence to Eq. [67].
Therefore, the average over all initial system states of the quadratic $S-T$ entanglement is just that of the generating unitary operator times $\frac{d_{S}}{d_{S}+1}$. It is first verified that if the operators $U_{t}$ form a complete orthogonal set, $E_{2}(\mathcal{W})=2\left(1-d_{S}^{-2}\right)$ is maximum and Eq. (67) yields $\left\langle E_{2}(S, T)\right\rangle=2\left(1-d_{S}^{-1}\right)$, the maximum attainable value in a $d_{S}$ dimensional space, entailing it is always maximum, irrespective of the initial state (sec. ПB).

In general, for a reduced set of $d$ orthogonal unitaries $U_{t}$, with $N=d \leq d_{S}^{2}, E_{2}(\mathcal{W})=2\left(1-d^{-1}\right)$ and hence

$$
\left\langle E_{2}(S, T)\right\rangle=2 \frac{d_{S}(d-1)}{d\left(d_{S}+1\right)} .
$$

In order to visualize this relation we define the effective average number of orthogonal states the system visits as

$$
\bar{d}_{S, T}=\frac{1}{1-\frac{1}{2}\left\langle E_{2}(S, T)\right\rangle}=\frac{d\left(d_{S}+1\right)}{d_{S}+d},
$$

such that $\left\langle E_{2}(S, T)\right\rangle=2\left(1-\frac{1}{\bar{d}_{S, T}}\right)$. If $d=d_{S}^{2}, \bar{d}_{S, T}=d_{S}$ becomes maximum, while if $d=d_{S}$, which is, for instance, the case of a constant Hamiltonian with spectrum $2 \pi k / N\left(d_{S}\right.$ orthogonal operators $\left.U_{t}=\exp [-i H t]\right)$, Eq. (74) leads to $\bar{d}_{S, T}=\left(d_{S}+1\right) / 2$, i.e., just half the maximum value for large $d_{S}$. For any other spectrum and $N=d_{S}, \bar{d}_{S, T} \leq\left(d_{S}+1\right) / 2$, i.e.,

$$
\left\langle E_{2}(S, T)\right\rangle \leq 2 \frac{d_{S}-1}{d_{S}+1} \quad\left(U_{t}=e^{-i H t}, \quad N=d_{S}\right) .
$$

Noticeably, it is sufficient to have $d \propto d_{S}\left(\ll d_{S}^{2}\right.$ for large $\left.d_{S}\right)$ to reach a high $\bar{d}_{S, T}$, i.e., $\bar{d}_{S, T}=\frac{m}{m+1}\left(d_{S}+1\right)$ if $d=m d_{S}$ (and $\left.m \leq d_{S}\right)$, as seen from (74).

\section{Measuring operator overlaps}

The overlaps $\left\langle U_{t} \mid U_{t}^{\prime}\right\rangle$, which are the operator fidelities defined in [43] and are involved in the quadratic entanglement (61) of the generating operator $\mathcal{W}$, can be experimentally obtained by measuring $\left|T_{t}\right\rangle\left\langle T_{t^{\prime}}\right|$ in the time part $T$ (Fig. 4). Remarkably, it is sufficient to start with the system in a maximally mixed state: If we trace out system $S^{\prime}$ in the operator history state (60), we obtain

$$
\rho_{S T}=\frac{1}{N d_{S}} \sum_{t, t^{\prime}} U_{t} U_{t^{\prime}}^{\dagger} \otimes|t\rangle\left\langle t^{\prime}\right|
$$

where we have written $\left|T_{t}\right\rangle$ as $|t\rangle$. Hence, tracing over $S$,

$$
\rho_{T}=\frac{1}{N} \sum_{t, t^{\prime}}\left\langle U_{t^{\prime}} \mid U_{t}\right\rangle|t\rangle\left\langle t^{\prime}\right|
$$

Thus, setting $U_{t, t^{\prime}}=U_{t} U_{t^{\prime}}^{\dagger}$,

$$
\left\langle\mid t^{\prime}\right\rangle\langle t \mid\rangle=\frac{1}{N}\left\langle U_{t^{\prime}} \mid U_{t}\right\rangle=\frac{1}{N d_{S}} \operatorname{Tr}\left[U_{t^{\prime}}^{\dagger} U_{t}\right]=\frac{1}{N d_{S}} \operatorname{Tr}\left[U_{t, t^{\prime}}\right] .
$$


Using again $\sigma_{t^{\prime} t}^{x}=\left|t^{\prime}\right\rangle\langle t|+| t\rangle\left\langle t^{\prime}\right|, \sigma_{t^{\prime} t}^{y}=-i\left(\left|t^{\prime}\right\rangle\langle t|-| t\rangle\left\langle t^{\prime}\right|\right)$, the trace of the evolution operator between any two times can then be obtained by measuring the averages of $\sigma_{t t^{\prime}}^{x}$ and $\sigma_{t^{\prime} t}^{y}$, which provide the real and imaginary parts:

$$
\left\langle\sigma_{t^{\prime} t}^{x}\right\rangle=\frac{2}{N} \operatorname{Re}\left[\left\langle U_{t} \mid U_{t^{\prime}}\right\rangle\right], \quad\left\langle\sigma_{t^{\prime} t}^{y}\right\rangle=\frac{2}{N} \operatorname{Im}\left[\left\langle U_{t} \mid U_{t^{\prime}}\right\rangle\right] .
$$

Of course, the state (76)) can be generated just by preparing system $S$ in the maximally mixed state, as the purifying system $S^{\prime}$ of the original operator state is traced out. Note also that $U_{0}=\mathbb{1}$, so that the averages $\left\langle\sigma_{0 t}^{\mu}\right\rangle$ determine $\operatorname{Tr} U_{t}$.

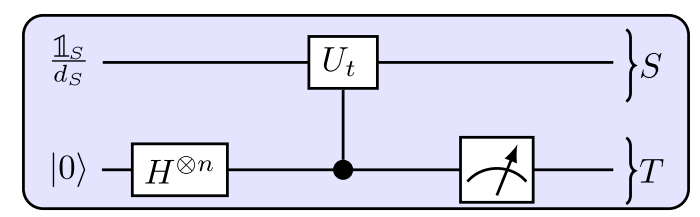

FIG. 4. (Color online) Schematic circuit representing the measurement of the operator overlaps (79).

In the special case $N=2, T$ is a single qubit and we recover the standard DQC1 scheme for measuring the trace of an operator 29]. The ensuing operator history state is $|\mathcal{W}\rangle=\frac{1}{\sqrt{2}}\left(\left|U_{0}\right\rangle\left|T_{0}\right\rangle+\left|U_{1}\right\rangle\left|T_{1}\right\rangle\right)$, and its quadratic entanglement is

$$
E_{2}(\mathcal{W})=1-\left|\left\langle U_{0} \mid U_{1}\right\rangle\right|^{2}=1-|\operatorname{Tr} U|^{2} / d_{S}^{2} .
$$

Its square root is just the entangling power of the $\mathrm{DQC1}$ circuit defined in 44].

\section{CONCLUSIONS}

Quantum mechanics has mostly considered time as an external classical parameter. In this work we have determined some fundamental properties concerning the generation and entanglement of discrete history states within a parallel-in-time discrete model of quantum evolution, based on a finite dimensional quantum clock 7]. It was first shown that a general unitary evolution for the system states follows from a static eigenvalue equation, which can be recast as a generalized discrete version of a Wheeler-DeWitt equation. The ensuing system-time entanglement is a measure of the actual number of distinguishable states visited by the system at distinguishable times and satisfies an entropic energytime uncertainty relation. Its dependence on the initial system state becomes attenuated for non-constant non-commuting Hamiltonians, and in particular we have presented a simple two-clock scheme which generates a maximally entangled history state irrespective of the seed state. Thus, history states essentially independent of initial conditions can be generated. On the other hand, for any fixed seed system state there is always a special clock basis selection for which the evolution corresponds to a constant Hamiltonian.

We have also shown that the quadratic entropy provides a convenient measure of the system-time entanglement entropy. It can be evaluated analytically and satisfies strict and physical upper and lower bounds, the former connected with the energy spread of the initial state and the latter determined by the evolution along the geodesic path between the initial and final states. Hence, such path, which provides the minimum evolution time 39], minimizes as well the quadratic $S-T$ entanglement entropy.

Finally, by means of the channel-state duality we have shown that the unitary operator generating the history state corresponds to an operator history state, with its quadratic entanglement entropy representing its entangling power. We have also provided a simple scheme which allows to efficiently obtain the overlaps between system states and the traces of the evolution operator between any two-times through measurements on the clock.

The present formalism is interesting as a fundamental aspect of quantum theory, where there are some possible scenarios to explore further in connection with quantum gravity, such as interaction between relational clocks 17, 18] and emergence of causality 45, 46]. The incorporation of time in a discrete quantum clock system also enables the development of new models of parallel-in-time simulation, taking advantage of the quantum features of superposition and entanglement. This description of time could be also suitable for application in Floquet systems, and in particular Floquet time crystals 47.

\section{APPENDIX}

Proof of the lower bound of Eq. 41). We first assume a sufficiently short final time $t_{f}$ such that $\left|\frac{\left(E_{k}-E_{k^{\prime}}\right) t_{f}}{2}\right| \leq \pi$ $\forall k \neq k^{\prime}$. Note that the overlap $\left|\left\langle S_{0} \mid S_{t_{f}}\right\rangle\right|$, Eq. (42), is unaffected by any translation $E_{k} \rightarrow E_{k}+2 j \pi / t_{f} \forall j \in \mathbb{Z}$, for a given $k$. The angle $\phi \in[0, \pi / 2]$ determined by this overlap can also be rewritten as

$$
\begin{aligned}
\phi & =\arcsin \sqrt{1-\left|\left\langle S_{0} \mid S_{t_{f}}\right\rangle\right|^{2}} \\
& =\arcsin \sqrt{2 \sum_{k \neq k^{\prime}}\left|c_{k} c_{k^{\prime}}\right|^{2} \sin ^{2} \frac{\left(E_{k}-E_{k^{\prime}}\right) t_{f}}{2}} .
\end{aligned}
$$

It is now expected that the overlap between any pair of intermediate states will be smaller than those between states $\left|S_{t}^{\min }\right\rangle=e^{-i H_{\min } t}\left|S_{0}\right\rangle$ along the geodesic, such that (Eq. (45)) $\left|\left\langle S_{t} \mid S_{t^{\prime}}\right\rangle\right| \leq\left|\left\langle S_{t}^{\min } \mid S_{t^{\prime}}^{\min }\right\rangle\right|=\left|\cos \left[\phi \frac{t-t^{\prime}}{t_{f}}\right]\right|$. This inequality is verified since the function

$$
F(s)=\arcsin \sqrt{2 \sum_{k \neq k^{\prime}}\left|c_{k} c_{k^{\prime}}\right|^{2} \sin ^{2} \frac{\left(E_{k}-E_{k^{\prime}}\right) t_{f} s}{2}}-\phi s
$$

where $s=\left|\frac{t-t^{\prime}}{t_{f}}\right| \leq 1$, is a concave function of $s$ for $s \in[0,1]$ and satisfies $F(0)=F(1)=0$, so that $F(s) \geq 0 \forall s \in[0,1]$. Hence, for short times $t_{f}$ such 
that all relative phases have yet not completed one period $\left(\left|E_{k}-E_{k^{\prime}}\right| t_{f}<2 \pi \forall k, k^{\prime}\right)$, all intermediate overlaps of the actual evolution are smaller than those along the geodesic, and hence the actual $E_{2}(S, T)$ entropy is larger than that along the geodesic path.

For larger times $t_{f}$, the inequality (41) also holds but for a different reason: If $\left|\frac{\left(E_{k}-E_{k^{\prime}}\right) t_{f}}{2}\right|>\pi$ for some pairs $k, k^{\prime}, F(s)$ may not be concave and can also be negative for some values of $s$. However, the relevant term of the exact expression for $E_{2}(S, T)$ satisfies

$$
\frac{\sin ^{2} \frac{\gamma N}{N-1}}{N^{2} \sin ^{2} \frac{\gamma}{N-1}} \leq \frac{\sin ^{2} \frac{(\gamma-j \pi) N}{N-1}}{N^{2} \sin ^{2} \frac{(\gamma-j \pi)}{N-1}}
$$

where $\gamma=\frac{\left(E_{k}-E_{k^{\prime}}\right) t_{f}}{2}$ and $j$ is such that $|\gamma-j \pi| \epsilon$
$[0, \pi / 2]$. This translation of the energy difference does not affect the overlap (Eq. (82)), but shows that the actual entropy $E_{2}(S, T)$ for large times will not become lower than the bound previously obtained. In this case some relative phases may have completed one or more periods, but the final effect will be to decrease the average overlap and hence to increase $E_{2}(S, T)$.

\section{ACKNOWLEDGMENTS}

The authors acknowledge support from CIC (RR) and CONICET (AB) of Argentina, and CONICET Grant PIP 112201501-00732.
[1] D.N. Page, W.K. Wootters, Phys. Rev. D 27, 2885 (1983); W. Wootters, Int. J. Theor. Phys. 23, 701 (1984).

[2] R. Gambini, R.A. Porto, J. Pullin, S. Torterolo, Phys. Rev. D 79, 041501(R) (2009).

[3] V. Giovannetti, S. Lloyd, L. Maccone, Phys. Rev. D 92, $045033(2015)$.

[4] E. Moreva, G. Brida, M. Gramegna, V. Giovannetti, L. Maccone, M. Genovese, Phys. Rev. A 89, 052122 (2014); E. Moreva, M. Gramegna, G. Brida, L. Maccone, M. Genovese, Phys. Rev. D 96, 102005 (2017).

[5] S. Massar, P. Spindel, A.F. Varon, C. Wunderlich, Phys. Rev. A 92, 030102(R) (2015).

[6] J.R. McClean, J.A. Parkhill, A. Aspuru-Guzik, Proc. Natl. Ac. Sci. U.S.A. 110, E3901 (2013); J.R. McClean, A. Aspuru-Guzik, Phys. Rev. A 91, 012311 (2015).

[7] A. Boette, R. Rossignoli, N. Gigena, M. Cerezo, Phys. Rev. A 93, 062127 (2016).

[8] P. Erker, M.T. Mitchison, R. Silva, M.P. Woods, N. Brunner, M. Huber, Phys. Rev. X 7, 031022 (2017).

[9] E.O. Dias, F. Parisio, Phys. Rev. A 95, 032133 (2017).

[10] A. Nikolova, G. K. Brennen, T.J. Osborne, G.J. Milburn, T.M. Stace, Phys. Rev. A 97, 030101(R) (2018).

[11] P.J. Coles, V. Katariya, S. Lloyd, I. Marvian, M.M. Wilde, arXiv:1805.07772 (2018).

[12] B.S. DeWitt, Phys. Rev. 160, 1113 (1967).

[13] C. Rovelli, Quantum Gravity, Cambridge (2004).

[14] K. V. Kuchar̃, Int. J. Mod. Phys. D 20, 3 (2011).

[15] C. J. Isham, in Integrable systems, quantum groups, and quantum field theory, L.A. Ibort, M.A. Rodrguez eds., Kluwer (Dordrecht), pp. 157, (1993).

[16] J. Tambornino, SIGMA 8, 017 (2012).

[17] M. Bojowald, P. A. Höhn, A. Tsobanjan, Phys. Rev. D 83, 125023 (2011).

[18] P. A. Höhn, E. Kubalova, A. Tsobanjan, Phys. Rev. D 86, 065014, (2012).

[19] J. Anandan, Y.Aharonov, Phys. Rev. Lett. 651697 (1990).

[20] P. Zanardi, C. Zalka, L. Faoro, Phys. Rev. A 62, 030301 (2000).

[21] M.A. Nielsen, C.M. Dawson, J.L. Dodd, A. Gilchrist, D. Mortimer, T.J. Osborne, M.J. Bremner, A.W. Harrow, A. Hines, Phys. Rev. A 67, 052301 (2003).

[22] T. Prosen, I. Piz̃orn, Phys. Rev. A 76, 032316 (2007).
[23] M. Musz, M. Kuś, K. Zyczkowski, Phys. Rev. A 87, 022111 (2013).

[24] A. Jamiołkowski, Rep. Math. Phys. 3, 275 (1972).

[25] M.D. Choi, Linear Algebra Appl. 10, 285 (1975).

[26] R.B. Griffiths, Phys. Rev. A 71, 042337 (2005).

[27] W. Dür, M. Hein, J.I. Cirac, and H.J. Briegel, Phys. Rev. A 72, 052326 (2005).

[28] M. Jiang, S. Luo, S. Fu, Phys. Rev. A 87, 022310 (2013).

[29] E. Knill, R. Laflamme, Phys. Rev. Lett. 81, 5672 (1998).

[30] A. Dembo, T.M. Cover, J.A. Thomas, IEEE Trans. on Inf. Th. 37, 1501 (1991).

[31] T. Przebinda, V. DeBrunner, M. Özaydin, IEEE Trans. on Inf. Th. 47, 2086 (2001); M. Özaydin, T. Przebinda, J. Func. An. 215, 241 (2004).

[32] L.I. Hirschman, Am. J. Math. 79, 152 (1957).

[33] H. Weyl, The Theory of Groups and Quantum Mechanics (Dover, New York), pp. 272-280 (1950).

[34] D. Galetti, A.F.R. De Toledo Piza, Physica A 149, 267282 (1988).

[35] A. Asadian, P. Erker, M. Huber, C. Klöckl, Phys. Rev. A 94, 010301(R) (2016).

[36] R. Bhatia, Matrix Analysis (Springer-Verlag, NY, 1997).

[37] R. Rossignoli, N. Canosa, Phys. Rev. A 67, 042302 (2003)

[38] H.P. Laba, V.M. Tkachuk, Cond. Matt. Phys. 20, 13003 (2017).

[39] K. Battacharyya, J. Phys. A 16, 2993 (1983); L. Mandelstam, I. Tamm, J. Phys. USSR 9, 249 (1945).

[40] D.C.l Brody, L.P.l Hughston, Phys. Rev. Lett. 77, 2851 (1996).

[41] W.K. Wootters, Phys. Rev. D 23, 357 (1981).

[42] M.J. Renes, R. Blume-Kohout, A.J. Scott, C.M. Caves, J. Math. Phys. 45, 2171 (2004).

[43] X.Wang, Z.Sun, Z.D. Wang, Phys. Rev. A 79,012105 (2009)

[44] C.S. Yu, X.X. Yi, H.S. Song, H. Fan, Phys. Rev. A 87, $022322(2013)$.

[45] M. Zych, F. Costa, I. Pikovski, Č. Brukner, Nat. Comm. 2, 505 (2011).

[46] E. Castro-Ruiz, F. Giacomini, Č. Brukner, PNAS 114, E2303 (2017).

[47] D.V. Else, B. Bauer, C. Nayak, Phys. Rev. Lett. 117, 090402 (2016). 